\title{
Effect of Educational Intervention on Improvement Of Quality of Life for Children with Epilepsy
}

\author{
Faten Shafik Mahmoud, \\ Lecturer of Pediatric Nursing, Faculty of Nursing Benha University, \\ Ismail Abou-El Ella Ramadan, \\ Professor of Pediatric Medicine, Faculty of Medicine, Benha University, \\ Mirret Mohammed Darouish \\ Professor of Pediatric Nursing, Faculty of Nursing, Cairo University, \\ Fatma Ahmed Ahmed Elsobkey \\ Assistant Lecturer of Pediatric Nursing, Faculty of Nursing Benha University,
}

\begin{abstract}
Epilepsy is one of a number of neurological disorders that affect quality of life. Nursing intervention of these children is crucial to maintaining their life and improving their quality of life. Therefore, the aim of this study was improve the quality of life for children with epilepsy, through, identifying children \& mother's knowledge and practice regarding epilepsy, identifying factors affecting on quality of life for epileptic child, designing and implementing a health educational intervention for mothers to improve quality of life for their children with epilepsy and evaluating the effect of educational intervention on mothers' knowledge and practice to improve quality of life of children with epilepsy. The research hypothesis Mothers after attending the educational intervention will show improvement on their children's quality of life. Setting, the study was conducted at the Student School Health Insurance in Benha. Sample, it involved all children with epilepsy (100) from both sexes, aged 6-15 year and their accompanying mothers recruited for the conduction of the study. The tools of data collection were 1) mothers and child assessment sheet 2) Epilepsy quality of life scale, 3) practice assessment sheet and 4) an educational program. The results showed significant improvement in mothers' knowledge and practice regarding caring for their children with epilepsy, also slightly improvement in children's quality of life after program implementation. The study concluded
\end{abstract}




\section{Tanta Scientific Nursing Journal}

that a success in improving the mothers' knowledge and practice regarding epilepsy. After three months of implementation the program the mothers' knowledge and practice was decline but still higher than before the program regarding all items of knowledge and practice, improving in all items of quality of life after 3 month of program implementation. The study recommended enhancing child's quality of life through periodical educational program and follow-up and regular educational programs at the out patient clinics about epilepsy and how to deal with epileptic child.

Key Words:- Epilepsy- Quality of life- Children.

\section{Introduction}

Epilepsy is a multifaceted chronic urinary and bowel incontinence (Ronen disorder which has diverse and complex effects on the overall wellbeing or subjective quality of life (QOL) of the children (Jude et al. 2009).

Epilepsy is the most common neurological disease worldwide and is second to stroke in causing neurological morbidity in developed countries. Approximately 45 to 100 million people worldwide are estimated to have active epilepsy (Fenichel, 2005).

Epilepsy is characterized by its episodic and chronic nature. The seizures usually produce brief periods of disruption, which include phenomena such as loss of consciousness, bodily distortion, injuries, unusual and often frightening psychological experiences as well as

et al., 2005). Bauder et al. (2007) stated that, seizures constitute the commonest neurological problem in children with significant epilepsy having its onset in childhood. Johnston (2008) added that, clinical classification of seizures may be difficult because the manifestation of different seizures may be similar. An electroencephalogram is a useful adjuvant to the classification of epilepsy because of the variability of seizure expressivity in this group.

Quality of life is a general term applied to the totality of physical, psychological and social functions. It reflects a general sense of happiness and satisfaction with our lives and environment. Generally, quality of life encompasses all aspects 
of life, including health, recreation, cultural rights, values, beliefs, aspirations and conditions that support a life containing these elements (U.S. Department of Health \& Human Services, 2005).

According to Epilepsy Wong (2005), families are having difficulties coping with epilepsy, interventions are essential to improve the outcome for the entire family. Education helps to eliminate the stigmas associated with epilepsy and may help to improve family functioning. Information is an important component to help parents, the child and siblings come to terms with the diagnosis of epilepsy.

Persons with childhood onset epilepsy are at a high risk for poor psychosocial outcomes, even without experiencing co-morbidities. The goal of management of children with epilepsy should be to enable the child and the family to lead a life as free as possible from the medical and psychosocial complications of epilepsy (Berto, 2009).

The nurse can play a vital role to improve quality of life of children suffering from epilepsy through counseling the children and their family to resolve any previously unidentified problems. These nursing efforts can assist with child compliance for therapy and result in better long term management of epilepsy with better seizures control and reduced adverse effects of medications that affect quality of life of children suffering from epilepsy (Christy and Jon, 2006).

\section{Significance of the problem}

Epilepsy becomes a common problematic neurological disorder in childhood. Seizures and epilepsy affect infants and children more than any other age group. Epilepsy is about twice as common in children as in adults (about 700 per 100,000 in children under the age of 16 years compared to 330 per 100,000 in adults). The incidence of status epileptics in developed countries is between 17 and 23/100,000 with a higher incidence in younger children (Neville et al., 2010).

\section{Aim of the study}

The aim of the study is to improve the 
quality of life for children with epilepsy, through:

1- Identifying children and mothers' knowledge and practice regarding epilepsy

2- Identifying factors affecting on quality of life for epileptic child.

3- Designing and implementing a health educational intervention for mothers to improve quality of life for their children with epilepsy.

4- Evaluating the effect of the educational intervention on mothers' knowledge and practice to improve quality of life of their children with epilepsy.

\section{Hypothesis}

Mothers after attending the educational intervention will show improvement on their children's quality of life.

\section{Subjects and methods}

The aim of the study is to improve the quality of life for children with epilepsy.

Subjects and methods of the current study were discussed under the following four designs:

1- Technical design.

2- Operational design.
3- Administrative design.

4- Statistical design.

\section{1- Technical design.}

The technical design includes the research design, setting, subjects, as well as tools of data collection.

\section{Research design:}

A quasi- experimental design was used in carrying out the study, with pre- post assessment of the impact of the intervention.

\section{Settings:}

This study was conducted at the Student School Health Insurance in Benha, which is the only established known place in Kaliubia that provides care to such children.

\section{Subjects:}

A sample of convenience 100 mothers and their children with epilepsy from both sexes, aged 6-15 year and their accompanying mothers recruited for the conduction of the study.

Tools and Technique of Data Collection:

Data collection was done through the following tools:

1- Questionnaire format: 
It was designed by the researcher under the guidance of supervisors, written in Arabic language and composed of two parts in the form of open ended and closed questions, it includes

\section{Part (1):}

a. Characteristics of the children as age, educational level and gender.

b. Past history of the children, as previous hospitalization and previous illness.

c. Present history of the disease, as age during the first seizures, how much seizures occur and complication of epilepsy.

d. Characteristics of the mother and family, as age, occupation, and education.

Part (2): It covered the following items.

a. Mothers' knowledge regarding epilepsy as concept, causes, and clinical manifestation.

b. Knowledge regarding practice of the mothers with children during seizures

c. Seizure severity questionnaire about events before, during, and after typical seizures.

Scoring system:

A scoring system was followed to obtain the outcome of mothers' knowledge.

- The total score of questionnaire responses was 30, accordingly, more than $75 \%$ was considered good knowledge, $60-<75$ was considered average knowledge and less than $60 \%$ was considered poor knowledge

\section{2- Practice Assessment Sheet:}

Designed by the researcher under the guidance of supervisors, it aimed at assessing the practice of the mothers toward the seizures. It was presented in (3) scales according to mothers' responses. Their practice was divided into either competent or incompetent, it included good, average, and poor.

\section{4- Epilepsy Quality of Life Scale:}

5- Adopted from Cramer (2003) it was modified by the researcher under the guidance of supervisors, to assess the impact of epilepsy on quality of life. It includes daily activity, mental activity, medication 
effect, seizures' worry, and overall quality of life.

\section{4- Educational program:}

It was designed by the researcher under the guidance of supervisors after reviewing of the related literature and according to need of children and their mothers.

\section{Proposed management program}

This program was designed to enhance quality of life for children with epilepsy through improving mothers' knowledge and practice regarding to caring of their children. It was designed in the form of handouts. It consists of providing the mothers with the essential information about epilepsy (definition, causes, signs \& symptoms, first aid during seizures and how to deal with the child during seizures).

\section{Program implementation}

The program was conducted through five sessions the time of each session was be verified 60 minutes according to the mothers' \& children's needs and condition. At the beginning of the first session, mothers were oriented regarding the program content, its purpose, and its impact on their children's condition. Mothers were informed about the time of the next session at the end of the setting.

Each session was started by a summary about what has been discussed in the previous one and presenting the objectives of the new session, using simple Arabic language, also the session ended by a summary of its content and feedback from the mothers was obtained to ensure that they got the maximum benefit.

\section{Program evaluation}

An effect of the program on children's quality of life was done through comparing the pre and post assessment scores of the mothers regarding their knowledge and practice toward seizures of their children, and quality of life. This evaluator phase was taken two times; an evaluation taken immediately after finish the implementation phase, then after three months, the second evaluation was done to assess retention of knowledge gained.

\section{II- Operational design.}

\section{Preoperational phase:}

An official letter was issued from the 
Faculty of Nursing to Health Insurance school administrators explaining the aim of the study for approval to conduct the study.

\section{Pilot study:}

A pilot study was carried out during March 2010; it involved 10 mothers and their children to test the applicability of the study tools and suitability of the selected setting. Accordingly, the necessary modifications were done in form of addition of some questions. Necessary changes as revealed from pilot study were performed as adding questions about effect of antiepileptic drugs. Mothers and children involved in the pilot were excluded from the study sample.

\section{Field work:}

The actual field work was carried out on end of March 2010 up to end December 2010. The average time needed for the completion of each questionnaire was around 45- 60 minutes. The researcher attends the study setting during morning. Each mother and his/her childe were individually interviewed using the questionnaire, practice sheet, and quality of life sheet. The researcher started to collect data and knowledge about mothers and their children after explaining the objectives of the study. She started each phase of the educational program with a summary for the previous one. The educational program plan was applied on 4 phases through demonstration and practices. Each phase required between 30-45 minutes for continuation of the plan. Teaching sessions were conducted three days / week. They were carried out using different teaching strategies, (lectures, group discussion, and role play).

\section{a. First Phase:}

This phase included assessment of the knowledge and practice of the mothers about epilepsy and children quality of life through using the developed tools as a pre-test

\section{b. Second phase:}

This phase included analysis of the pretest findings and identification of mothers' knowledge and practice toward epilepsy issues, then the epilepsy educational program was designed. 
c. Third phase :( program planning \&implementation):

Based on the results from the interviewing questionnaire and the quality of life questionnaire as well as literature review, and educational health program regarding the epilepsy issues was developed by the researcher.

\section{General Objectives}

By the end of the implementation of the epilepsy educational program, mothers of children with epilepsy will be to have adequate and proper knowledge and practice toward epilepsy and care for their children.

\section{Specific Objectives:-}

By the end of the program, the mothers were able to:

Identify concept of epilepsy.

Mention causes, and signs and symptoms of seizures.

Describe epilepsy and impact on quality of life.

Apply first aid during seizures

\section{d. Fourth phase:}

Follow up of the program was carried out after 3 months using pre/ post-tests.

\section{Ethical and Legal considerations:}

The aim of the study was explained to each subject before applying the tools to gain confidence and trust.

An oral consent was obtained from each subject and his / her mother participating in the program, informing them that they have the right to withdraw at any time without giving any reason.

The study was conducted in a safe place for the children and mothers.

Data were collected and treated confidentially.

\section{III- Administrative Design:}

An official approval was obtained from the administrators of the study setting to carry out the study. A clear explanation was given about the nature, importance and expected outcomes of the study.

\section{IV- Statistical Design:}

The collected data were organized, categorized, tabulated and analyzed. Data were presented in the tables using descriptive statistics in the form of frequencies, percentages, mean scores, standard deviations and other statistical 
tests as Coefficient correlation test and paired sample $\mathrm{T}$ test.

\section{Results}

As regard the socio-demographic characteristics of mothers with children having epilepsy. The mean age of mothers was $34.00 \pm 6.09$ years. Regarding family history for epilepsy, $56 \%$ of them have no history for epilepsy while $44 \%$ have. Of those who have history $31 \%$ their relatives were having epilepsy, as well $13 \%$ of their siblings, $67.0 \%$ of parents were related to each others and $33 \%$ were not.

The mean age of children was $11.390 \pm$ 2.1969 years, regarding child's sex $69 \%$ of them were males, and more than half of them $(54 \%)$ were the first child in the family. This table also shows that, $80 \%$ of children from preparatory schools.

The majorities of children have signs and symptoms of seizures, have flushed face then refuse to eat before convulsions. Most of them have abnormal actions and movements during seizures, and have sever impact on their lives, with mental psychological and physical impact.
Table (1) :this table showed that there were a highly significant improvement in knowledge mean scores of mothers regarding the epilepsy items during immediate post test and three months after test as compared to pre program mean scores $\mathrm{p}(<0.001)$.

Table (2): This table revealed that, the children total knowledge score was improved overall items after implementing the program and decline after three months of implementing the program. Although the children score knowledge was decline after three months of program, the improvement was observed more than pre- program. Moreover there was a highly statistical significant differences was observed regarding total knowledge for children pre, post, and after three months of program $\mathrm{P}<0.001$

Table (3): this table showed that there were a highly significant improvement in practice mean scores of mothers regarding the epilepsy items during immediate post test and Three months after test as compared to pre program mean scores $\mathrm{p}(<0.001)$. 
This table (4): shows that there were statistically significant improvements in QOL of children with epilepsy mean scores regarding to physical wellbeing after three month test as compared to pre program mean scores $(\mathrm{p}<0.001)$.

This table 5 pointed that there were highly statistically significant improvements on quality of life mean scores for children with epilepsy regarding overall quality of life domain,, after three months test as compared to pre program $(<0.001)$.

This table 6 reveals that, there is highly statistically significant difference between pre, post immediately and after three month program implementation in relation to mothers' knowledge, where about $99 \%$ of them before program had poor knowledge, while immediately post program their knowledge improved to be good for $93 \%$ after three months, $76 \%$ of them were good in their knowledge $(\mathrm{X} 2=286.10 \mathrm{P}=0.001)$.

This table 7 shows that $24 \%$ of the studied mothers have incompetent practice in preprogram, while $100 \%$, $95 \%$ of them have competent practice immediately post and after three months $(\mathrm{X} 2=179.26, \mathrm{P}=<0.001)$.

Table (8): shows that $48 \%$ of the studied children had poor quality of life in preprogram, while $92 \%$ of them have good quality of life after three month (X2=170.96, $\mathrm{P}<0.001)$.

This table 9 reveals that there were highly statistically significant correlations $(r=.291$ and $.460 \quad \mathrm{P}<0.01)$ between age of the studied mothers and their total practice throughout preprogram and after three month of program implementation. Meanwhile there was no statistical significant correlation immediately post of program implementation.

This table 10 indicates that there was a highly statistically significant correlation $(\mathrm{r}=\quad .327, \quad .257 \quad \mathrm{P}<0.01)$ between age of the studied children and their total quality of life through out program phase. This table also shows that there was a highly significant correlation $(\mathrm{r}=\quad .280, \quad \mathrm{P}=<0 \quad .001)$ between educational level of the studied children and their total quality of life throughout preprogram phases. 


\section{Tanta Scientific Nursing Journal}

Meanwhile there was no statistically significant correlation between educational level and total quality of life after three month of program implementation $(\mathrm{r}=0.61, \mathrm{P}=>0.005)$.

This table 11 shows that there were highly statistically significant correlations $(\mathrm{r}=0.474,0.356$, at $\mathrm{P}=<0$ .001) between age of children and their total knowledge score preprogram phase and immediately post. As regards educational level, there were highly significant correlation $(r=0.471, \quad 0.400$ at $\mathrm{P}=<0 \quad .001)$ between children's educational level and their knowledge preprogram phase and immediately after program. Meanwhile there was no a statistically significant correlation $(\mathrm{r}=$ $0.174 \mathrm{P}=>0.005)$ between education and children knowledge after three month of program implementation. 
Table (1): Mean score of mothers' knowledge regarding Epilepsy before, immediately after, and after three months

\begin{tabular}{|c|c|c|c|c|c|c|c|c|}
\hline \multirow{2}{*}{$\begin{array}{c}\text { Knowledge } \\
\text { Item }\end{array}$} & \multicolumn{2}{|c|}{ Mean \pm SD } & \multirow[b]{2}{*}{$\begin{array}{c}\mathbf{T} \\
\text { test }\end{array}$} & \multirow[b]{2}{*}{$\begin{array}{c}\mathbf{P} \\
\text { value }\end{array}$} & \multicolumn{2}{|c|}{ Mean \pm SD } & \multirow[b]{2}{*}{$\begin{array}{c}\mathbf{T} \\
\text { test }\end{array}$} & \multirow[b]{2}{*}{$\begin{array}{c}\mathbf{P} \\
\text { value }\end{array}$} \\
\hline & Before & $\begin{array}{c}\text { Immediately } \\
\text { after }\end{array}$ & & & Before & $\begin{array}{c}\text { After } \\
\text { three } \\
\text { months }\end{array}$ & & \\
\hline $\begin{array}{l}\text { Definition of } \\
\text { epilepsy }\end{array}$ & $\begin{array}{c}0.12 \\
\pm 0.62\end{array}$ & $3.87 \pm 0.48$ & $\begin{array}{c}- \\
45.67\end{array}$ & $<0.001$ & $\begin{array}{c}0.12 \\
\pm 0.62\end{array}$ & $3.31 \pm 1.33$ & -22.15 & $<0.001$ \\
\hline $\begin{array}{l}\text { Causes of } \\
\text { epilepsy }\end{array}$ & $\begin{array}{c}0.250 \pm 0 \\
.97\end{array}$ & $5.63 \pm 0.94$ & $\begin{array}{c}- \\
36.98\end{array}$ & $<0.001$ & $\begin{array}{c}0.250 \pm 0 \\
.97\end{array}$ & $\begin{array}{l}4.66 \\
\pm 1.85\end{array}$ & -21.46 & $<0.001$ \\
\hline Clinical picture & $\begin{array}{c}0.25 \\
\pm 0.89\end{array}$ & $4.69 \pm 0.76$ & $\begin{array}{c}- \\
37.79\end{array}$ & $<0.001$ & $\begin{array}{c}0.25 \\
\pm 0.89\end{array}$ & $\begin{array}{c}4.05 \\
\pm 1.58\end{array}$ & -21.61 & $<0.001$ \\
\hline $\begin{array}{l}\text { Types of } \\
\text { epilepsy }\end{array}$ & $\begin{array}{c}0.17 \\
\pm .55\end{array}$ & $2.93 \pm 0.32$ & 44.41 & $<0.001$ & $\begin{array}{l}0.17 \\
\pm .55\end{array}$ & $\begin{array}{c}2.72 \pm \\
0.69\end{array}$ & -27.24 & $<0.001$ \\
\hline $\begin{array}{l}\text { Definition of } \\
\text { Status } \\
\text { epileptics }\end{array}$ & $0.28 \pm 0.64$ & $2.94 \pm 0.28$ & $\begin{array}{c}- \\
38.03\end{array}$ & $<0.001$ & $0.28 \pm 0.64$ & $\begin{array}{c}2.65 \pm \\
0.84\end{array}$ & -23.20 & $<0.001$ \\
\hline $\begin{array}{l}\text { Characteristics } \\
\text { of Status } \\
\text { epileptics }\end{array}$ & $\begin{array}{c}0.12 \pm \\
0.54\end{array}$ & $3.95 \pm 0.22$ & $\begin{array}{c}- \\
56.12\end{array}$ & $<0.001$ & $\begin{array}{c}0.12 \pm \\
0.54\end{array}$ & $\begin{array}{c}3.62 \pm \\
0.89\end{array}$ & -35.00 & $<0.001$ \\
\hline $\begin{array}{l}\text { Predisposing } \\
\text { Factors that } \\
\text { cause seizures }\end{array}$ & $\begin{array}{c}0.30 \pm 0 . \\
91\end{array}$ & $4.86 \pm 0.48$ & $\begin{array}{c}- \\
37.99\end{array}$ & $<0.001$ & $\begin{array}{c}0.30 \pm 0 . \\
92\end{array}$ & $\begin{array}{c}4.29 \pm \\
1.15\end{array}$ & -27.33 & $<0.001$ \\
\hline
\end{tabular}

$\mathrm{T}=$ paired $\mathrm{T}$ test

P highly Significant at $<0.001$

Table (2): Mean score of children's' knowledge regarding Epilepsy before, immediately after, and after three months

\begin{tabular}{|c|c|c|c|c|c|c|c|c|}
\hline \multirow{2}{*}{$\begin{array}{l}\text { Knowledge } \\
\text { Item }\end{array}$} & \multicolumn{2}{|c|}{ Mean \pm SD } & \multirow{2}{*}{$\begin{array}{l}\text { T } \\
\text { Test }\end{array}$} & \multirow{2}{*}{$\begin{array}{l}\mathbf{P} \\
\text { value }\end{array}$} & \multicolumn{2}{|c|}{ Mean \pm SD } & \multirow{2}{*}{$\begin{array}{l}T \\
\text { test }\end{array}$} & \multirow{2}{*}{$\begin{array}{l}\mathbf{P} \\
\text { value }\end{array}$} \\
\hline & Before & $\begin{array}{l}\text { Immediately } \\
\text { After }\end{array}$ & & & Before & $\begin{array}{l}\text { After } \\
\text { three } \\
\text { months }\end{array}$ & & \\
\hline $\begin{array}{l}\text { Definition of } \\
\text { epilepsy }\end{array}$ & $\begin{array}{c}0.72 \\
\pm 1.22\end{array}$ & $3.87 \pm 0.48$ & -23.57 & $<0.001$ & $\begin{array}{c}0 . \vee r \\
\pm 1.22\end{array}$ & $3.14 \pm 1.29$ & $\begin{array}{c}- \\
14.54\end{array}$ & $<0.001$ \\
\hline $\begin{array}{ll}\begin{array}{l}\text { Causes } \\
\text { epilepsy }\end{array} & \text { of } \\
\end{array}$ & $\begin{array}{c}0.94 \pm \\
1.692 \\
\end{array}$ & $5.63 \pm .94$ & -24.96 & $<0.001$ & $\begin{array}{c}0.94 \pm \\
1.692 \\
\end{array}$ & $4.34 \pm 1.77$ & $\begin{array}{c}- \\
14.37 \\
\end{array}$ & $<0.001$ \\
\hline
\end{tabular}

Vol. 5 No. 2 November, 2013 


\section{Tanta Scientific Nursing Journal}

\begin{tabular}{|c|c|c|c|c|c|c|c|c|}
\hline $\begin{array}{l}\text { Clinical } \\
\text { picture }\end{array}$ & $\begin{array}{c}0.54 \\
\pm .1 .04\end{array}$ & $4.69 \pm .76$ & -22.29 & 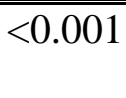 & $\begin{array}{c}0.54 \\
\pm .1 .04\end{array}$ & $3.57 \pm 1.74$ & $\begin{array}{c}- \\
16.28\end{array}$ & $<<0.001$ \\
\hline $\begin{array}{l}\text { Types } \\
\text { epilepsy }\end{array}$ & $0.440 \pm .78$ & $2.93 \pm .32$ & -30.73 & $<0.001$ & $0.440 \pm .78$ & $2.33 \pm 0.96$ & $\begin{array}{c}- \\
15.60\end{array}$ & $<0.001$ \\
\hline $\begin{array}{l}\text { Definition of } \\
\text { Status } \\
\text { epileptics }\end{array}$ & $0.53 \pm .93$ & $2.94 \pm 0.28$ & -25.25 & $<0.001$ & $0.53 \pm .93$ & $\begin{array}{c}2.37 \\
\pm 0.99\end{array}$ & $\begin{array}{c}- \\
13.02\end{array}$ & $<0.001$ \\
\hline $\begin{array}{l}\text { Characteristics } \\
\text { of Status } \\
\text { epileptics }\end{array}$ & $\begin{array}{c}0.39 \pm \\
.79\end{array}$ & $3.95 \pm 0.22$ & $\begin{array}{c}- \\
40.461\end{array}$ & $<0.001$ & $\begin{array}{c}0.39 \pm \\
.79\end{array}$ & $3.21 \pm 1.24$ & $\begin{array}{c}- \\
19.42\end{array}$ & $<0.001$ \\
\hline $\begin{array}{l}\text { Predisposing } \\
\text { Factors }\end{array}$ & $\begin{array}{c}1.14 \pm \\
1.65 \\
\end{array}$ & $4.86 \pm 0.48$ & $\begin{array}{c}- \\
21.017 \\
\end{array}$ & $<0.001$ & $\begin{array}{c}1.14 \pm \\
1.65 \\
\end{array}$ & $\begin{array}{l}3.75 \pm \\
1.45\end{array}$ & $\begin{array}{c}- \\
12.30 \\
\end{array}$ & $<0.001$ \\
\hline
\end{tabular}

$\mathrm{T}=$ paired $\mathrm{T}$ test

P highly Significant at $<0.001$

Table (3): Mean score of practice of the mothers toward seizures

\begin{tabular}{|c|c|c|c|c|c|c|c|c|}
\hline \multirow{2}{*}{\begin{tabular}{|l|} 
Practice \\
Item
\end{tabular}} & \multicolumn{2}{|l|}{ Mean \pm SD } & \multirow{2}{*}{$\begin{array}{l}\text { T } \\
\text { Test }\end{array}$} & \multirow{2}{*}{$\begin{array}{l}P \\
\text { value }\end{array}$} & \multicolumn{2}{|c|}{ Mean \pm SD } & \multirow{2}{*}{\begin{tabular}{|l|}
$T$ \\
test
\end{tabular}} & \multirow{2}{*}{$\begin{array}{l}P \\
\text { value }\end{array}$} \\
\hline & Before & $\begin{array}{l}\text { Immediately } \\
\text { After }\end{array}$ & & & Before & $\begin{array}{l}\text { After } \\
\text { three } \\
\text { months }\end{array}$ & & \\
\hline $\begin{array}{l}\text { Remove any } \\
\text { furniture }\end{array}$ & $1.45 \pm 0.70$ & $2.95 \pm 0.26$ & $-\overline{20.50}$ & $<0.01$ & $1.45 \pm 0.70$ & $\begin{array}{l}2.81 \pm \cdot \\
.53\end{array}$ & $\begin{array}{l}- \\
15.83\end{array}$ & $<0.001$ \\
\hline $\begin{array}{l}\text { Place the child } \\
\text { on his side }\end{array}$ & $\begin{array}{l}1.44 \\
\pm 0.74\end{array}$ & $2.97 \pm 0.17$ & $\begin{array}{l}- \\
19.19\end{array}$ & $<0.01$ & \begin{tabular}{|l|}
1.44 \\
\pm 0.74 \\
\end{tabular} & $\begin{array}{l}2.78 \pm \\
0.52\end{array}$ & $\begin{array}{l}- \\
14.02\end{array}$ & $<0.001$ \\
\hline Rise bed side & $\begin{array}{l}1.49 \\
\pm 0.74\end{array}$ & $3.00 \pm 0.00$ & $\begin{array}{l}- \\
20.26\end{array}$ & $<0.01$ & \begin{tabular}{|l|}
1.49 \\
\pm 0.74
\end{tabular} & $\begin{array}{l}2.71 \pm \\
0.62\end{array}$ & $\begin{array}{l}- \\
11.31\end{array}$ & $<0.001$ \\
\hline $\begin{array}{l}\text { Remove any } \\
\text { clothing in the } \\
\text { neck }\end{array}$ & $\begin{array}{l}1.62 \pm \\
0.86\end{array}$ & $2.96 \pm 0.19$ & $\begin{array}{l}- \\
14.18\end{array}$ & $<0.01$ & \begin{tabular}{|l|}
$1.62 \pm$ \\
0.86
\end{tabular} & $\begin{array}{l}2.79 \pm \\
0.54\end{array}$ & $\begin{array}{l}- \\
12.13\end{array}$ & $<0.001$ \\
\hline \begin{tabular}{|l|} 
Attempt stop \\
seizures
\end{tabular} & $\begin{array}{l}1.92 \\
\pm 3.0^{\circ}\end{array}$ & $2.98 \pm 0.14$ & -3.47 & $<0.001$ & $\begin{array}{l}1.92 \\
\pm 3.0^{\circ}\end{array}$ & $2.69 \pm 0.53$ & \begin{tabular}{|l|}
-2.42 \\
\end{tabular} & $<0.005$ \\
\hline $\begin{array}{l}\text { Remove any } \\
\text { thing in the } \\
\text { child mouth }\end{array}$ & $\begin{array}{l}1.57 \\
\pm 0.81\end{array}$ & $3.00 \pm 0.00$ & $\begin{array}{l}- \\
17.71\end{array}$ & $<0.001$ & \begin{tabular}{|l|}
1.57 \\
\pm 0.81
\end{tabular} & $\begin{array}{l}2.82 \\
\pm 0.48\end{array}$ & $\begin{array}{l}- \\
13.84\end{array}$ & $<0.001$ \\
\hline \begin{tabular}{|l|} 
Give child \\
medication \\
during seizures \\
\end{tabular} & $\begin{array}{l}1.72 \\
\pm .88\end{array}$ & $2.95 \pm 0.2 \Upsilon$ & $\begin{array}{l}- \\
12.78\end{array}$ & $<0.001$ & $\begin{array}{l}1.72 \\
\pm .88\end{array}$ & $\begin{array}{l}2.69 \pm \\
0.68\end{array}$ & \begin{tabular}{|l|}
-7.29 \\
\end{tabular} & $<0.005$ \\
\hline
\end{tabular}

Vol. 5 No. 2 November, 2013 


\begin{tabular}{|c|c|c|c|c|c|c|c|c|}
\hline \multirow{2}{*}{$\begin{array}{l}\text { Practice } \\
\text { Item }\end{array}$} & \multicolumn{2}{|c|}{ Mean \pm SD } & \multirow{2}{*}{$\begin{array}{l}\mathbf{T} \\
\text { Test }\end{array}$} & \multirow{2}{*}{$\begin{array}{l}\mathbf{P} \\
\text { value }\end{array}$} & \multicolumn{2}{|c|}{ Mean \pm SD } & \multirow{2}{*}{$\begin{array}{l}T \\
\text { test }\end{array}$} & \multirow{2}{*}{$\begin{array}{l}P \\
\text { value }\end{array}$} \\
\hline & Before & $\begin{array}{l}\text { Immediately } \\
\text { After }\end{array}$ & & & Before & $\begin{array}{l}\text { After } \\
\text { three } \\
\text { months }\end{array}$ & & \\
\hline $\begin{array}{l}\text { Remain with } \\
\text { the child until } \\
\text { conscious }\end{array}$ & $\begin{array}{l}2.38 \\
\pm .80\end{array}$ & $3.00 \pm 0.00$ & -7.79 & $<0.001$ & $\begin{array}{l}2.38 \\
\pm .80\end{array}$ & $\begin{array}{l}2.76 \\
\pm 0.60\end{array}$ & -4.29 & $<0.005$ \\
\hline $\begin{array}{l}\text { Go to critical } \\
\text { care }\end{array}$ & $\begin{array}{l}1.61 \\
\pm 0.78 \\
\end{array}$ & $3.00 \pm 0.00$ & $\begin{array}{l}- \\
17.89 \\
\end{array}$ & $<0.001$ & $\begin{array}{l}1.61 \\
\pm 0.78 \\
\end{array}$ & $\begin{array}{l}2.80 \\
\pm 0.49 \\
\end{array}$ & $\begin{array}{l}- \\
13.82 \\
\end{array}$ & $<0.001$ \\
\hline
\end{tabular}

$\mathrm{T}=$ paired $\mathrm{T}$ test

P highly Significant at $<0.001$

Table (4): Mean scores of physical wellbeing domain of QOL among children before program, and after three months.

\begin{tabular}{|c|c|c|c|c|}
\hline \multirow[b]{2}{*}{$\begin{array}{l}\text { physical wellbeing } \\
\text { Items. }\end{array}$} & \multicolumn{2}{|c|}{ Mean \pm SD } & \multirow{2}{*}{$\begin{array}{c}\text { T- } \\
\text { test }\end{array}$} & \multirow{2}{*}{$\begin{array}{c}\text { P- } \\
\text { value }\end{array}$} \\
\hline & Before & After three months & & \\
\hline Fatigue & $7.60 \pm 1.62$ & $4.98 \pm 1.75$ & 15.26 & $<0.001$ \\
\hline Appetite changes & $6.90 \pm 1.50$ & $3.08 \pm 1.34$ & 20.01 & $<0.001$ \\
\hline Sleep changes & $7.33 \pm 1.57$ & $2.54 \pm 1.70$ & 19.54 & $<0.001$ \\
\hline Shortness /difficult in breathing & $6.82 \pm 1.69$ & $3.07 \pm 1.82$ & 13.69 & $<0.001$ \\
\hline headache & $8.08 \pm 1.35$ & $3.80 \pm 1.97$ & 19.23 & $<0.001$ \\
\hline Nausea / vomiting & $7.08 \pm 1.57$ & $3.20 \pm 1.39$ & 16.51 & $<0.001$ \\
\hline pain in hand / feet & $6.10 \pm 1.72$ & $4.03 \pm 1.52$ & 9.31 & $<0.001$ \\
\hline Mouth dryness & $6.47 \pm 1.86$ & $2.99 \pm 1.85$ & 15.92 & $<0.001$ \\
\hline Jump changes & $5.89 \pm 2.06$ & $2.75 \pm 1.63$ & 12.10 & $<0.001$ \\
\hline pain in stomach & $5.93 \pm 2.08$ & $2.82 \pm 1.44$ & 10.94 & $<0.001$ \\
\hline
\end{tabular}

Vol. 5 No. 2 November, 2013 
Table (5): Mean score of effect of quality of life among children under study before and after three months.

\begin{tabular}{|c|c|c|c|c|}
\hline \multirow[b]{2}{*}{ Quality of life item } & \multicolumn{2}{|c|}{ Mean \pm SD } & \multirow{2}{*}{$\begin{array}{c}\mathrm{T} \\
\text { test }\end{array}$} & \multirow{2}{*}{$\begin{array}{c}\mathbf{P} \\
\text { value }\end{array}$} \\
\hline & Before & $\begin{array}{c}\text { After three } \\
\text { months }\end{array}$ & & \\
\hline Quality of life and feeling of satisfaction & $6.22 \pm 2.69$ & $4.04 \pm 2.20$ & 9.32 & $<0.001$ \\
\hline QOL and degree of distress & $6.88 \pm 2.83$ & $4.44 \pm 1.89$ & 10.89 & $<0.001$ \\
\hline Your evaluation for the QOL & $6.89 \pm 2.78$ & $3.27 \pm 1.77$ & 13.13 & $<0.001$ \\
\hline
\end{tabular}

Table (6): Relation between mothers' knowledge throughout program phases.

\begin{tabular}{|c|c|c|c|c|c|c|c|c|}
\hline \multirow{3}{*}{ Items } & \multicolumn{8}{|c|}{ Total knowledge } \\
\hline & \multicolumn{2}{|c|}{ poor } & \multicolumn{2}{|c|}{ Average } & \multicolumn{2}{|c|}{ Good } & \multirow[t]{2}{*}{$\mathbf{X}^{2}$} & \multirow[t]{2}{*}{ P value } \\
\hline & $\mathbf{N}$ & $\%$ & $\mathbf{N}$ & $\%$ & $\mathbf{N}$ & $\%$ & & \\
\hline Pr-program & 99 & 99.0 & 1 & 1.0 & 0 & 0.0 & \multirow{3}{*}{286.10} & \multirow{3}{*}{$<0.001$} \\
\hline Immediately- post & 2 & 2.0 & 5 & 5.0 & 93 & $93 \%$ & & \\
\hline After three month & 4 & 4.0 & 19 & 19.0 & 76 & $76 \%$ & & \\
\hline
\end{tabular}


Table (7): Relation between mother total practices throughout program phases.

\begin{tabular}{|c|c|c|c|c|c|c|}
\hline \multirow{3}{*}{\begin{tabular}{|c|} 
Items \\
Pre program
\end{tabular}} & \multicolumn{6}{|c|}{ Total practice } \\
\hline & \multicolumn{2}{|c|}{ Competent } & \multicolumn{2}{|c|}{ incompetent } & \multirow{2}{*}{$\mathbf{X}^{2}$} & \multirow{2}{*}{$P$ value } \\
\hline & 24 & 24.0 & 76 & 76.0 & & \\
\hline Immediately -post & 99 & 99.0 & 1 & 1.0 & \multirow[t]{2}{*}{179.26} & \multirow[t]{2}{*}{$<0.001$} \\
\hline After three month & 95 & 95.0 & 5 & 5.0 & & \\
\hline
\end{tabular}

Table (8): Relation between quality of life for children throughout the program phases.

\begin{tabular}{|c|c|c|c|c|c|c|c|c|}
\hline \multirow[b]{3}{*}{ Items } & \multicolumn{8}{|c|}{ Total quality of life } \\
\hline & \multicolumn{2}{|c|}{ Poor } & \multicolumn{2}{|c|}{ Average } & \multicolumn{2}{|c|}{ Good } & \multirow[b]{2}{*}{$\mathrm{X} 2$} & \multirow[b]{2}{*}{$P$ value } \\
\hline & $\mathbf{N}$ & $\%$ & $\mathbf{N}$ & $\%$ & $\mathbf{N}$ & $\%$ & & \\
\hline Pre-Program & 48 & 48.0 & 52 & 52.0 & 0 & 0.0 & & \\
\hline $\begin{array}{ll}\text { After } & \text { three } \\
\text { month } & \end{array}$ & 6 & 6.0 & 2 & 2.0 & 92 & 92.0 & 170.96 & $<0.001$ \\
\hline
\end{tabular}


Table (9): Correlation between mother's age and total practice throughout program phases.

\begin{tabular}{|c|c|c|}
\hline \multirow{2}{*}{$\begin{array}{c}\text { Items } \\
\text { total practice }\end{array}$} & \multicolumn{2}{|c|}{ Age of the mothers } \\
\hline & $\mathbf{R}$ & P value \\
\hline Pre-program & 0.291 & $<0.001$ \\
\hline Immediately post & 0.34 & $>0.05$ \\
\hline After three month & 0.460 & $<0.001$ \\
\hline
\end{tabular}

Table (10): Correlation between children age \& educational level and their total quality of life throughout program phases

\begin{tabular}{|l|c|c|c|c|}
\hline \multirow{2}{*}{$\begin{array}{c}\text { Total Quality of } \\
\text { Life }\end{array}$} & \multicolumn{2}{|c|}{ Children's age } & \multicolumn{2}{c|}{ Children's educational } \\
\cline { 2 - 5 } & r & p & r & p \\
\hline Pre-program & 0.327 & $<0.001$ & 0.280 & $<0.001$ \\
\hline $\begin{array}{l}\text { After three } \\
\text { month }\end{array}$ & 0.257 & $<0.001$ & 0.061 & $>0.005$ \\
\hline
\end{tabular}

Vol. 5 No. 2 November, 2013 
Table (11) Correlation between children's age \& educational level and their total knowledge throughout program phases.

\begin{tabular}{|c|c|c|c|c|}
\hline \multirow{2}{*}{ Total Knowledge } & \multicolumn{2}{|c|}{ children's } & \multicolumn{2}{|c|}{ Education } \\
\hline & $\mathbf{R}$ & $\mathbf{P}$ & $\mathbf{r}$ & $\mathbf{P}$ \\
\hline Pre-program & 0.474 & $<0.001$ & 0.471 & $<0.001$ \\
\hline Immediately post & 0.356 & $<0.001$ & 0.400 & $<0.001$ \\
\hline After three month & 0.218 & $<0.001$ & 0.174 & $>0.005$ \\
\hline
\end{tabular}

\section{Discussion}

Epilepsy is a chronic condition with numerous social and psychological consequences. Epilepsy is the occurrence of sporadic electrical storms in the brain commonly called seizures. These storms cause behavioral manifestations such as staring and/or involuntary movements such as grand mal seizures (Wolters, \& Kluwer

\section{Health 2009).}

Investigating study finding regarding to the frequency of epileptic seizures it was obvious that, more than half of studied children have epileptic seizures every day, this may be due to the severity of the cases and that almost two thirds of children under study aren't compliant with antiepileptic drugs. In relation to complying with medical follow up, the present study showed that more than two thirds of studied children were not attending to the Students' School Health Insurance for medical follow up. According to the researcher point of view this finding may be due to illiteracy of the parents and lack of knowledge about the 
importance of regular follow up. As regards the adverse side effects of antiepileptic drugs, the results of the present study showed that about two thirds of the studied children suffering from epilepsy had severe effect from antiepileptic drugs. This finding agreed with Gouda (2005), who found that, the majority of the children suffering from epilepsy had adverse effects from antiepileptic drugs. This finding goes also in line with a recent study carried out by Devinsky et al., (2010), who reported that, most types of antiepileptic drugs produce severe side effects for a high percentage of children having epilepsy. This study result clarified that, the majority of children have signs and symptoms of seizures (aura).

As regards mental change that occur after seizures (post-ictal phase), the current study result showed that the majority of children have mental impact, after an epileptic seizure. In a similar study done by, So et al. (2010) who stated that, in the post-ictal phase, some cases suffer from reduced consciousness and altered behavior. This may consist of aggression and inappropriate behavior. Some children may be confused after a seizure and they may even run out into the street in their night clothes. Post ictal psychosis, which is characterized by well-systematized delusions and hallucinations in a setting of preserved orientation and alertness. Correct medical treatment should usually limit or prevent mental reactions after seizures.

On assessing mothers' knowledge regarding epilepsy, before the program implementation the majority of them had unknown knowledge and they cannot clarify all items of epilepsy, but after implementation of the program the majority of them were able to identify the all items of epilepsy. This could be due to mothers' need for simple information to understand their children's illness and how to deal with it. This finding was in agreement with Elsayed (2003), and Karande et al. (2007), who emphasized that mothers' knowledge before program about concept of epilepsy cannot be compared by their knowledge after program.

Before program implementation the educated mothers showed more information and had positive attitude than the illiterate ones, while after program implementation the educated and illiterate mothers had gained knowledge well and they did care for 
their children as they learned through the program, but the educated mothers responded to the researcher more easily than the illiterate ones. This finding is in agreement with $\mathrm{El}$ sayed, (2003) and Hafz (2004), who mentioned that, it is well known that the educated mothers are able to take care of their children especially with epilepsy, children who need more care and understanding of their needs and problems. It has been reported that education and cultural level have an effect on children's condition both during disease and during wellness.

This study result revealed that the majority of mothers had incompetent practice during seizures for their children with epilepsy; this may be due to the parents' anxiety during the seizure as most of them believe that their children are dying during a seizure. in a similar study, Huang, et al. (2006) stated that, the educational level and economic status are important variables affecting practice of parents toward epilepsy. They added that parents, whether experienced or not, should be provided enough information at every visit because parents experience anxiety and fear during every seizure. This was supported by Zeglam (2009), who reported that, the practice of mothers before and after the introduction of the information change significantly.

Regarding physical wellbeing items of quality of life, the finding of this study revealed that, there were highly significant improvements in all items after program implementation compared with before program. The Epilepsy Foundation (2011) mentioned that, $46 \%$ of the respondents with epilepsy described their overall health as "fair" or "poor" compared to $18.5 \%$ of those who did not have epilepsy. Considering child's fatigue, the present study finding revealed that slightly less than three quarters of studied children have severe impact in pre program as compared to post program, where nearly half of them have mild impact. This study result agreed with that of Hamelin et al. (2010), who reported in their study about fatigue in epilepsy that most of the sample exhibit high chronic fatigue scores and also fatigue represents a further complication of epilepsy, associated with depression. The impact of fatigue should be considered with the aim of improving quality of life and care of children with 
epilepsy. in a similar study, Frucht et al. (2006) mentioned that, the feeling of fatigue, with premature tiredness during mental activity, and lack of energy, is common in children with epilepsy. Fatigue is also commonly reported as a factor that can provoke seizures.

The present study result revealed that there was a significant improvement regarding appetite changes in post program as compared to pre program implementation. According to the Epilepsy Foundation (2011) a loss of appetite and subsequent weight loss are side effects of some antiepileptic medications. A child with epilepsy loses weight; this may be due to a low mood which can sometimes result in a decreased appetite.

The current study result showed that, more than half of the studied children have sever impact related to sleep change in pre program phase as compared to post program phase, where almost three quarters of them have mild impact. Sleep is especially important in epilepsy. Herman et al. (2001) stated that, most types of seizures are affected by sleep, although the degree varies widely from type to type and from childe to child. The effects of seizures and seizure medication on the quality of sleep can make the relationship.

Regarding to social activities of daily living wellbeing domain items of QOL there were highly significant improvements in all items after three months of program compared with before program implementation.

A study carried out by Bell, (2008), stated that, children with epilepsy have a high risk for change in activities of daily living as they may lack confidence to pursue social and recreational activities due to fear of their seizures and the stigma associated with epilepsy.

The present study showed that, nearly three quarters of studied children had severe impact related to illness or treatment interference with activities at school in preprogram phase, this may be due to fear from having a seizure during activity. This finding was in agreement with Rutter et al. (2008), who mentioned that, although most children with epilepsy attend school but they can not participate in all activities. Some may need to take medicine at school, as they may need help with certain subjects, or extra time on tests. They may sometimes have 
seizures at school.

The present study revealed that, nearly the majority of studied children have sever impact related to isolation in preprogram phase as compared to post program phase where more than two third of them have mild impact. This may be due to the effectiveness of the educational program on children. In their study, Jacoby and Austin (2007) mentioned that, many children with epilepsy hide their condition from others, which can result in social isolation and reduced self esteem and can lead to a feeling of helplessness and depression. They added children with epilepsy often feel a sense of social isolation and have an overall reduced quality of life. Social isolation and quality of life have also been shown to be significantly compromised in caregivers of children with epilepsy.

As regards interference illness or treatment with the study, most of the study children have severe impact in preprogram phase as compared to post program phase where approximately two third of them have mild impact, this may be due to seizures and medications which affect children's learning disabilities and behavior problems in learning. This result agreed with U.S. Department of Education, (2009) who stated that, children with epilepsy may have learning disabilities and behavior problems. Children of average or above average intelligence, and lived with epilepsy, had at least a two-year deficit in reading skills. This significantly lower educational performance could be attributed to cognitive impairment caused by the etiology of epilepsy itself, the frequency of seizure activity and the effects of AED.

The present study result revealed that there was a significant improvement regarding mental and emotional wellbeing, and self esteem items of quality of life post three months of program implementation. This result is supported with Soyupek et al. (2010) who stated that, mental and cognitive disturbances are relatively common in epilepsy, especially refractory epilepsy. However the previous result disagreed with Russo et al. (2008), who mentioned that children with epilepsy experience reduced quality of life and self-concept compared with typically developing peers.

The finding of the present study illustrated that, the majority of children have a problem 


\section{Tanta Scientific Nursing Journal}

in learning in preprogram phase as compared to post program implementation more than two third of them have mild impact in learning. This is agreement with Global Campaign Against Epilepsy (2008), which stated that, often children with epilepsy receive inadequate schooling and deficit in reading skills. This significantly lower educational performance could be attributed to cognitive impairment caused by the etiology of epilepsy itself, the frequency of seizure activity and the effects of AED therapy in children with epilepsy. Cognitive impairment can take various forms but the most common are memory impairments, mental slowing and attention deficits.

In a study entitled" A resource for teachers' epilepsy" Little (2002) reported that, many children with epilepsy (20-50\%) will experience learning difficulties as children with epilepsy had high level of under achievement and made less progress than would be expected for their age and intelligence level, due to episodic nature of epilepsy.

The present study clarified that more than half of studied children have moderate impact related to depression in preprogram phase. This finding is supported by Science Daily (2010), which mentioned that, researchers have noted a higher incidence of depression among children with epilepsy than the general population or others with chronic conditions such as diabetes, for a long time, depression was thought to be a complication of epilepsy. In children with refractory epilepsy, the presence of depression is one of the most important variables to have an impact on their quality of life, even more than the seizure frequency and severity (Mendez et al., 2010).

As regards memory troubles in children with epilepsy, the present study result denoted that the majority of studied children have severe impact in preprogram phase as compared to post program implementation approximately three quarters of them have mild impact. In this respect, Little (2002) mentioned that, memory is a natural brain process that allows people to retain the things that they have learned. Seizures affect memory by interfering with attention, which affects the person's ability to input and retrieve information.

The present study finding revealed that more than half of studied children have severe 
impact related to drug and its effect on QOL. This may be due to that those children didn't follow physician's prescription related to drug therapy and were irregular in taking these drugs. In this respect, Mula et al. (2010) stated that, many of AEDs can cause symptoms of attention deficit hyperactivity disorder, depression and anxiety in children. As well the Epilepsy.com Staff (2010) added that, many children worry that the medication they take for their epilepsy causes poor memory. The side effects of some medications (e.g. poor concentration and drowsiness) can affect remembering. However, their memory will be improved if the drugs are stopping or lowering the number of seizures their have.

The present study revealed that there was significant improvements in items of seizure effects on QOL of children under study after three months of program implementation as compared to preprogram. This is in agreement with Kay (2011), who stated that, if the seizures are frequent, they can affect children's QOL, clarifying that, an individual with frequent seizures may be socially stigmatized and unable to participate in certain activities because of his condition. in similar study, Lorin et al. (2004) demonstrated that, although QOL has multiple determinants, seizure worries are the most important factors affecting QOL in children with intractable epilepsy. As well Johnson et al. (2005) pointed that frequent, severe, and chronic seizures reduce QOL, but are less powerful predictors of QOL than anxiety and depression.

Regarding the relationship between mothers' knowledge and their level of education, in the current study result, showed that before the program implementation there was a significant difference between illiterate and educated mothers while this difference disappeared after program implementation. This result was supported by Abdullah (2010), who found in her study that there were a positive correlation between mothers' knowledge and their level of education preprogram phase.

The present study showed that there was a significant difference between mothers' knowledge and their occupation throughout program phases, It obvious that, employed mothers have higher knowledge than housewife mothers, which may be attributed to social interaction and its effect on 
mothers' knowledge.

The current study also revealed that there was a positive correlation between children's age and their total QOL. This finding was highly supported by Miller et al. (2003,) who reported that age difference exists below 13 years' children that indicate significantly higher than in older children. The researcher believes that the children at adolescent years are more sensitive to their illness and may be affected by the community view to epilepsy as stigma.

As regards child's level of education and its relation to QOL the present study denoted that, there was a highly positive correlation which is in accordance with Devinsky et al. (2010), who found a positive correlation between level of education and QOL of children suffering from epilepsy.

\section{Conclusion}

It can be concluded from the present study that:

The mothers' knowledge and practice related to epilepsy was deficient as none of them pre-program had good knowledge or competent practice. The program succeeded in improving the mothers' knowledge and practice regarding to epilepsy. As regards
QOL of children, by comparing the results in the pre- and after three months of program implementation, they showed improvements in all domains of the QOL; in relation to physical, psychological, mental, social and activities of daily living.

\section{Recommendations}

\section{Based on the findings and conclusion of the current study, the following are the main suggested recommendations:}

-Provide mothers of children with epilepsy by updated pamphlets, posters and an Arabic booklet about epilepsy, which contain an action plan suitable for children's nature in order to facilitate improving mother's knowledge as they considered the main member in children's care plan

- Periodical educational training program for mothers caring for their children having epilepsy to improve their QOL.

- Further studies can be carried out on specific areas of epilepsy and its management, such as the effect of ketogenic diet on seizure's control.

\section{References}

1- Bauder, F., Wohlrab, G., \& Schmitt, B. Neonatal seizures: Eyes open or closed. Epilepsia; 2007; 48: 394-396. 
2- Bell G.S. Drawing people with epilepsy: How great is the risk? Neurology Aug 2008; 19.17 (8): 578-820

3- Berto,P. Quality of life in patients with epilepsy and impact of treatments.: F. A. Davis Company: Philadelphia 2009;1039-1059.

4- Christy, L. \& Jon, B. Strategies and innovations for successful quality improvement in behavioral health. Journal of Nursing Care Quality;2006; 19 (3):197-206.

5- Cramer, Seizure severity questionnaire, available At: www.epilepsy.com Accessed on 1-6-2010

6- Devinsky, O., Westbrook, L., Cramer, J., Glassman , M., \& Camfield, C. Risk factors for poor health - related quality of life in adolescents with epilepsy. Epilepsia ;2010; 40:1715-1720.

7- Elsayed S.,F.: An educational program for caregivers of children with cerebral palsy. Doctorate Thesis, Faculty of Nursing, Pediatric Nursing, Ain Shams University,2003;.115-120.

8- Epilepsy Foundation. Depression \& Anxiety in Epilepsy, Journal of Neurology, Neurosurgery and
Psychiatry: Epilepsy Foundation.com. Accessed on 12-8-2010.

9- Epilepsy.com Staff. Effects on thinking. Seizures thinking (2n.d.). Available at: http://www.epilepsy.com/epilepsy/thinki ng, $\quad$ Retrieved in March 13, 2010

10- Fenichel,G.M. Paroxysmal disorders in: Fenichel GM (ed) clinical pediatric neurology $\left(4^{\text {th }}\right.$ ed.) W.B. Saunders Company, Philadelphiaq, USA2005;. 145.

\section{1- Frucht,M.M.,} Quigg,M., Schwaner,C., Fountain, N.BDistribution of seizure precipitants among epilepsy syndromes. Epilepsia; .2006; 41:1534-9.

\section{2-Global Campaign Against Epilepsy}

Out of the shadows. Available at: www.who.int/mental_health/manageme nt/en/GcaeBroEn.pdf accessed on 29 April 2010.

13- Gouda,S. Factors affecting quality of life of epileptic children. Master Thesis faculty of Nursing, Ain Shams University;2005; 108-115

14- Hafz,M.M. Nutritional rehabilitation 
for infant and children with cerebral palsy having feeding difficulties. Doctorate Thesis. Faculty of Nursing, Pediatric Nursing, Ain Shams University, . 2006; 140-146.

\section{5- Hamelin,S., Kahane,P., \& Vercueil,L.} Fatigue in epilepsy: a prospective, interictal and post-ictal survey; Epilepsy unit, Neurology department, Grenote University Hospital, Grenoble, France; 2010; 153-160.

16- Herman,S.T., Walczak,T.S, \& Bazil,C.W. : Distribution of partial seizures during the sleep-wake cycle: Differences by seizure onset site; Neurology;56;2001;:1453-1459, American Epilepsy Society. .

17-Huang, M.C., Huang,C.C, and Thomas, K. Febrile convulsions: Development and validation of a questionnaire to measure parental knowledge, attitudes, concerns and practices. J Formos Med Assoc $2006 ;. ; 105: 38-48$.

18-Jacoby，A. \& Austin， J.K: Social stigma for adults and children with epilepsy. Epilepsia.2007; 48(1 9):69.
19- Johnson EK, Jones JE, and Seindenberg M. 2005; The relative impact of anxiety, depression and clinical seizure features on healthrelated quality of life in epilepsy. Epilepsia 2005; 45:544-50.

20- Johnston,M.V., Seizures in childhood. In: Kliegman, Behrman and Jenson Nelson Textbook of Pediatrics $\left(18^{\text {th }}\right)$ ed., WB Saunders Company, Philadelphia, USA2008;. 2457-2476.

21- Jude,U. Ohaeri, Abdel,W. Awadalla, A. A. \& Farah. Quality of life in people with epilepsy and their family caregivers: An Arab experience using the short version of WHO Quality of Life Instrument, Quality of Life, V. 30; No.10; .2009; 1023-1030.

22- Karande S., Patil,S.,\& Kulkarni,M. (2007): Impact of an educational program on parental knowledge of cerebral palsy. Developmental Clinic, Division of Pediatric Neurology, Department of Pediatrics.

23-Kay, W. (2011): eHow Contributor Available at: the Effect of Seizures on the Brain eHow.com seizures-brain Accessed on 20-12-2010. 
24- Little, A. (2002): A resource for teachers' epilepsy Queensland .Available At: http://www. epilepsy Queensland . Accessed on 16-12-2010.

25-Lorin, D.W., Meador, K.J., \& Lee, G.P. (2004): Determinations of quality of life in epilepsy. Epilepsy Behavior; 5: 976-980.

26-Mendez， M.F., Cummings， J.L.,\& Benson,D.F. Depression in epilepsy. Significance and phenomenology. Arch Neurol.; 2010;43(8):766-70.

27- Miller,V., Palermo,T.M.,\& Grewe,S.D Quality of life in pediatric epilepsy: Demographic and disease-related predictors and comparison with healthy controls. Epilepsy Behavior;2003; 4:3642

28- Mula,M. Trimble, M.R., YuenA., Liu,R.S., and Sander, J.W Psychiatric adverse events during levetiracetam therapy. Neurology. 2010;9; 61(5):704-6.

29- Neville BG, Chin RF, and Scott RC;(

30- Childhood convulsive status epilepticus: epidemiology, management and outcome. Acta Neurol Scand Suppl. 2007;186:21-4. [abstract]

31- Ronen, G.M., Streiner, D.L.,
Rosenbaum, P., \& the Canadian Pediatric Epilepsy Network. (2005): Health-related quality of life in children with epilepsy: Development and validation of self-report and parent proxy measures. J Personality Ass; 80:99-103.

32- Russo, R.N., Goodwin, E.J., Miller, M.D. Haan, E.A., Connell,T.M., \& Crotty,M.,(2008): Self-esteem, self concept and quality of life in children with hemiplegics cerebral palsy. Flinders University Rehabilitation Studies Unit, University of Adelaide, Adelaide, South Australia.

33- Rutter, M., Graham P., \& Yule, W., (2008): A neuropsychiatric study in child-hood. Clin Dev Med; 3:35-36.

34-Science- Daily. (2010): DC Epilepsy and depression: A two-Way street? Washington;.American Association For the Advancement of Science. Com. Accessed on13-12-2010.

35-Soyupek, F., Aktepe, E., Savas, S., \& Askin, A. (4Feb,2010): Do the selfconcept and quality of life decrease in $\mathrm{CP}$ patients? Focussing on the predictors of self-concept and quality of 
life. Department of Physical Medicine and Rehabilitation, Suleyman Demirel University, Isparta, Turkey. National Center for Biotechnology Information, U.S. National Library of Medicine. Rockville Pike, Bethesda MD, 20894 USA. 2009

36-So, N.K., Savard, G., Andermann, F., Olivier, A., \& Quesney, L.F. Acute postictal psychosis: a stereo EEG study. Epilepsia. Mar-Apr;2010; 31(2):188-93.

37-US Department of Health and Human Services. Healthy people Understanding and improving health, Jones and Bartlett Publishers: USA. 2005;1(11): 10 .

38- U.S. Department of Education National Center for Education Statistics Staff. The condition of education, Retrieved March 15, 2010 from http://nces.ed.gov/programs/coe/

39- Wolters Kluwer Health. Quality of life in patients with epilepsy and impact of treatments | Adis in its journal PharmacoEconomics. Accessed at 2009;16-12-2010.

40- Wong, L., Hockenberry, M., Wilson, D., Winkelstein, M., \& Schwartz, P.
Wong's essentials of pediatric nursing: $\left(6^{\text {th }}\right.$ ed.,) Mosby, London,2005;.10981106.

41-Zeglam A., (2009): Auditing the attitude and knowledge of parents of children with febrile seizures. Proceedings of 7 th all Libyan Pediatrics conference: Gharian, Libya African Journal of Neurological Science. 2009;29(1).

42- Abdullah M. Enhancing the quality of life for children with cerebral palsy at Benha City Doctorate (Thesis),Faculty of nursing, Benha University.2010 
Tanta Scientific Nursing Journal

Vol. 5 No. 2 November, 2013 
Tanta Scientific Nursing Journal

Vol. 5 No. 2 November, 2013 
Tanta Scientific Nursing Journal

Vol. 5 No. 2 November, 2013 